\title{
Review of: "Philip Morris International used the e- cigarette, or vaping, product use associated lung injury (EVALI) outbreak to market IQOS heated tobacco"
}

\author{
Moira Gilchrist ${ }^{1}$
}

1 PMI Science

Potential competing interests: The author is an employee of Philip Morris International

\section{Study alleging Philip Morris International used the EVALI outbreak to market IQOS requires substantial methodological revision and further peer review, or retraction}

\section{Rapid Response}

A brief review of this 'Industry Watch' article alleging heated tobacco product advertising through an earned media approach highlights significant methodological errors that are serious enough to invalidate the article's conclusions, including its title. The authors allege that Philip Morris International (PMI) used the e-cigarette, or vaping, product use associated lung injury (EVALI) outbreak to promote IQOS in September 2019 and the weeks that followed. Using the authors' own tool (TobaccoWatcher.org), we replicated their search strategy and revealed several fundamental and concerning errors in the authors' analysis.

They report a rise in news stories mentioning IQOS on and after $25^{\text {th }}$ September 2019, and falsely attribute this rise to an article published on our website on $24^{\text {th }}$ September 2019, which they also falsely describe as a "press release", despite it never being published through a press release distribution service. Our analysis shows that the authors failed to consider several confounding and unrelated events that caused the rise in news coverage of both IQOS and EVALI during the time period in question and which can be found by replicating the authors' search strategy in TobaccoWatcher.org.

For example, on $25^{\text {th }}$ September 2019, Philip Morris International (PMI) issued a single press release via Business Wire[1] entitled "Philip Morris International Inc. and Altria Group, Inc. End Merger Discussions" (PMI/Altria Announcement). The release noted that "the companies have agreed to focus on launching IQOS in the U.S." PMI is listed on the New York Stock Exchange and is therefore subject to the rules of the NYSE and the Securities and Exchange Commission, including 
requirements for the disclosure of material events such as the PMI/Altria Announcement. We estimate that during a date range similar to that used by the authors, of the total number of stories retrieved using the authors' search terms, $>85 \%$ were reporting on the above noted PMI/Altria Announcement. The authors failed to mention this and other confounders in their discussion.

The authors state that "the original PMI press release (sic) was republished (including editing or revisions to make the republication consistent with the publisher's format) in 14 additional news outlets." We performed a full text review of the 14 articles mentioned and of those that were still accessible, none mentioned IQOS. The authors' conclusion that "PMI capitalised on EVALI by using an earned news media strategy to market their IQOS brand of heated tobacco for free across scores of news outlets that reached millions of readers. This marks the first known case where a tobacco company used the EVALI outbreak to promote tobacco products" is therefore completely unsupported by their own data.

These facts, and other errors detected in this article, were communicated to the authors on $23^{\text {rd }}$ April 2021. In summary, we believe that the article is unreliable, and that urgent substantial revision or retraction is warranted to prevent other studies citing the currently published inaccuracies.

Note: submitted as a Rapid Response to Tobacco Control on 5th May, 2021

\section{[1] https://philipmorrisinternational.gcs-web.com/static-files/78a6afb3-107d-4e8f-ad11-} $\underline{b 66 e 94 b 8 a 4 b 8}$

A more comprehensive review of the Article is available in the attached Supplementary Data file. Concerns about the validity of the Article were raised with the Authors on 23rd April, 2021 by e-mail. To date, no response has been received from the Authors. The Editor-in-Chief of Tobacco Control was also made aware of the concerns on 5th May, 2021 by e-mail. Follow-up e-mails were sent to the Editor-in-Chief on (1) 18th May, 2021, (2) 8th June, 2021 and (3) 21st June, 2021. As of the date of this article, Tobacco Control have yet to publish the above Rapid Response. 